\title{
Granulomatosis with polyangitis (GPA) or ANCA associated vasculitis (AAV): a case report
}

\author{
Corresponding author: \\ Daniela Buklioska llievska, \\ Department of Pulmonology \\ and Allergology in General Hospital \\ „,8th September”, Skopje, Macedonia, \\ The Former Republic of Yugoslav, \\ e-mail: dbuklioska@yahoo.com
}

Medical Research Journal 2020; Volume 5, Number 4, 281-285 DOI: 10.5603/MRJ.a2020.0034 Copyright (C) 2020 Via Medica ISSN 2451-2591

\begin{abstract}
GPA is a systemic, necrotizing, small-vessel vasculitis associated with circulating anti-neutrophil cytoplasmic autoantibodies (ANCA), and so-called ANCA-associated vasculitis (AAV). A white woman in her early thirties was sent to the hospital because of hemoptysis, dyspnea, fever, cough, general fatigue, swollen joints. Chest radiography on hospital admission detected diffuse small nodules in both lungs. Laboratory tests: white blood cells $13.9 \ldots 16.7 \ldots 18.2 \times 10^{9} / \mathrm{L}$, hemoglobin $91 \ldots 80 \ldots 110 \mathrm{~g} / \mathrm{L}$, hematocrit $30 \ldots 25 . .35 \%$, erythrocytes $3.7 \ldots 3.1 \ldots 4.2 \times 10^{12} / \mathrm{L}$, platelet count $4.45 \ldots 5.5 \ldots 5.7 \times 10^{9} / \mathrm{L}$, sedimentation rate $72 \ldots 60 \ldots 45 \mathrm{~mm} / \mathrm{h}$ per the first hour, C-reactive protein $111 \ldots 80 \ldots 35 \mathrm{mg} / \mathrm{L}$, D-dimer $4100 \ldots 3500 \ldots 1250$ $\mathrm{ng} / \mathrm{ml}$. Biochemistry analysis: kidney function tests (blood urea nitrogen 10.4 ..7.4 ..5.6 mmol/L, creatinine $110 \ldots 95 \ldots 87 \mu \mathrm{mol} / \mathrm{L}$, liver tests normal, albumin $28 \mathrm{~g} / \mathrm{L}$, total protein $58 \mathrm{~g} / \mathrm{l}$. Urinalysis: mild proteinuria (+), 16-18 red blood cells, epithelial cells $(++), 24$-hour proteinuria 0,52g/L. Gas analyses: partial oxygen pressure $7.6 \ldots 8.9 \ldots 9.3 \mathrm{kPa}$, the partial pressure of carbon dioxide $3.7 \ldots 4.2 \ldots 4.6 \mathrm{kPa}$, oxygen saturation $90 \ldots 93 \ldots 95 \%$. She was febrile $38.5^{\circ} \mathrm{C}$, heart rate 122 beats/minute, swollen ankles, pale skin, conjunctival hyperemia, coagulated blood in both nostrils. Lung auscultation sounds normal. Lung CT scan detected diffuse, bilateral, small nodules, some of them with areas of cavitation and pseudo-cavitation. Autoimmune antibody tests: positive c-ANCA 95U/ml, negative p-ANCA, negative RF $158 \mathrm{IU} / \mathrm{ml}$, positive antiproteinase-3 $900 \mathrm{U} / \mathrm{ml}$. Renal ultrasonography normal. IL-6 serum level normal 5 pg/ml, also C3 level $1.2 \mathrm{~g} / \mathrm{L}$ and C4 level $0.3 \mathrm{~g} / \mathrm{L}$. Lung ultrasonography: bilateral, irregular, subpleural, hypoechogenic changes, with different size and central necrosis. Tracheobronchial mucosa was vulnerable, inflamed and edematous, bronchial lavage negative for malignancy and infection. Bronchial biopsy detected necrotic granulomas with multinucleated giant and inflammatory cells confirming the diagnosis of PGA. Cyclophosphamide combined with corticosteroids was given four months until the time of remission. The corticosteroid dose was slowly reduced and cyclophosphamide was switched to azathioprine to maintain remission. The treatment duration of the maintenance immunosuppressive medication after 10 months follow up is still ongoing with continuous monitoring of side effects.

Key words: ANCA associated vasculitis (AAV), granulomatosis with polyangiitis (GPA), lung nodules, autoimmune disease, ANCA, necrotizing vasculitis
\end{abstract}

Med Res J 2020; 5 (4): 281-285

\section{Introduction}

Vasculitides according to the size of vessel involvement are divided in large (giant cell arteritis), medium (polyarteritis nodosa) and small-vessel vasculitides (ANCA-associated vasculitides, AAV). Granulomatosis with polyangiitis (GPA), microscopic polyangiitis and eosinophilic granulomatosis with polyangiitis belong (EGPA) to AAV [1]. GPA is a systemic, necrotizing, small to medium-sized blood vessels, characterized by granulomatous inflammation, pauci-immune necrotizing glomerulonephritis, vasculitis, and an association with circulating anti-neutrophil cytoplasmic autoantibodies (ANCA), and is so-called AAV. Estimates of the overall incidence range between $0.5-20$ cases/million with a prevalence of 20-160 cases/million, dominantly in middle Europe and Australia/New Zealand, and relatively rare in non-Caucasian populations [2]. AAV is a chronic disease, and greater emphasis has turned to improving treatment-related toxicity, reducing relapses 
and providing alternative treatments for refractory disease. Since the introduction of cyclophosphamide and high-dose glucocorticoids in the 1970s, AAV has transformed from a disease with $80 \%$ mortality, into chronic relapsing and remitting disease with 10-20\% 1-year mortality. Current outcomes at 5 years are $25 \%$ mortality and a $50 \%$ relapse rate [3]. Nowadays, remission can be achieved and maintained in most cases with a combination of high-dose steroid and immunosuppressive drugs (methotrexate, azathioprine, cyclophosphamide, rituximab, tocilizumab, mofetil mycophenolate) [1, 4-5]. This therapy has to be continued for at least 24 months (3-6 months of remission induction therapy, followed by 12-24 months of remission maintenance therapy) after a substantial remission has been obtained because the early cessation of treatment is associated with an increased risk of relapse. For this reason, patients should be regularly monitored in order to promptly diagnose and treat a possible recurrence of AAV [3, 6]. Targeted immunotherapy is substantially improving the management of AAV [7]. Rituximab, an anti-CD20 B cell-depleting therapy, has been used for over a decade in patients with $\mathrm{AAV}$, offers a significant advance in the treatment of these diseases. It has an established role for remission induction and is now being investigated as a remission maintenance agent. In patients at risk of malignancy, women of childbearing potential, and patients previously treated with cyclophosphamide, rituximab may be considered preferable to cyclophosphamide, which has well-established malignancy risks. Also is more effective than cyclophosphamide for treating relapses. $[3,6]$. Treatment with tocilizumab was able to induce a complete and sustained disease remission in a patient with severe multisystemic MPA, as well as normalization of circulating levels of IL-6-associated pro-inflammatory cytokines and chemokines [5]. Maintenance treatment with the interleukin-5 antagonist, mepolizumab is effective in decreasing glucocorticoid requirements and in alleviating asthma and sinonasal symptoms, its efficacy on the vasculitis remains somewhat unclear. Omalizumab as a recombinant, humanized, a monoclonal antibody against human immunoglobulin $E$ have been reported to be efficacious in refractory asthma associated with EGPA. Belimumab, an anti-B lymphocyte stimulatory monoclonal antibody is in clinical trials for the management of GPA [7-9]. Immunoglobulins can be helpful as an adjuvant treatment for active AAV with severe immune depression, notably when infections occur. Plasma exchange is indicated in AAV with advanced renal dysfunction and, perhaps, in the event of alveolar hemorrhage. Plasmapheresis is effective for very severe disease, reducing dialysis dependence from 60 to $40 \%$ in the first year, but with no effect on mortality or long-term renal function, probably due to established renal damage $[7,10]$. Structured clinical assessment using the Birmingham Vasculitis Activity Score and Vasculitis Damage Index should form the basis of an individual treatment plan and personalize the care of AAV patients [10].

\section{Case presentation}

A white woman in her early thirties was sent to our hospital because of hemoptysis, shortness of breath, fever, cough, general fatigue, swollen joints. She claimed to be healthy until a few months ago when epistaxis and fever appeared. Laboratory and chest radiograph were normal in that period. Because of symptom progression, another chest radiography was performed and massive right paracardial consolidation was noted (Fig. 1). Routine blood work revealed: white blood cells $13.9 \ldots 16.7 \ldots 18.2 \times 10^{9} / \mathrm{L}$, hemoglobin $91 \ldots 80 \ldots 110 \mathrm{~g} / \mathrm{L}$, hematocrit $30 \ldots 25 . .35 \%$, erythrocytes $3.7 \ldots 3.1 \ldots 4.2 \times 10^{12} / \mathrm{L}$, platelet count $4.45 \ldots 5.5 \ldots 5.7 \times 10^{9} / \mathrm{L}$, sedimentation rate (ESR) was elevated $72 \ldots 60 \ldots 45 \mathrm{~mm} / \mathrm{h}$ per the first hour (ref. values $0-20 \mathrm{~mm}$ per hour), C-reactive protein (CRP) $111 \ldots 80 \ldots 35 \mathrm{mg} / \mathrm{L}$ (ref. range less than $3.03 \mathrm{mg} / \mathrm{L}$ ), D-dimer 4100...3500...1250 ng/ml (ref range $<500 \mathrm{ng} / \mathrm{ml}$ ). Biochemistry analysis: kidney function tests (blood urea nitrogen 10.4...7.4...5.6 mmol/ (normal values 2.8-7.2 mmol/L), creatinine 110...95 ..87 $\mu \mathrm{mol} / \mathrm{L}$ (ref. range $49-115 \mu \mathrm{mol} / \mathrm{L}$ ), liver function tests normal, albumin $28 \mathrm{~g} / \mathrm{L}$ (ref range $34-50 \mathrm{~g} / \mathrm{L}$ ), total protein

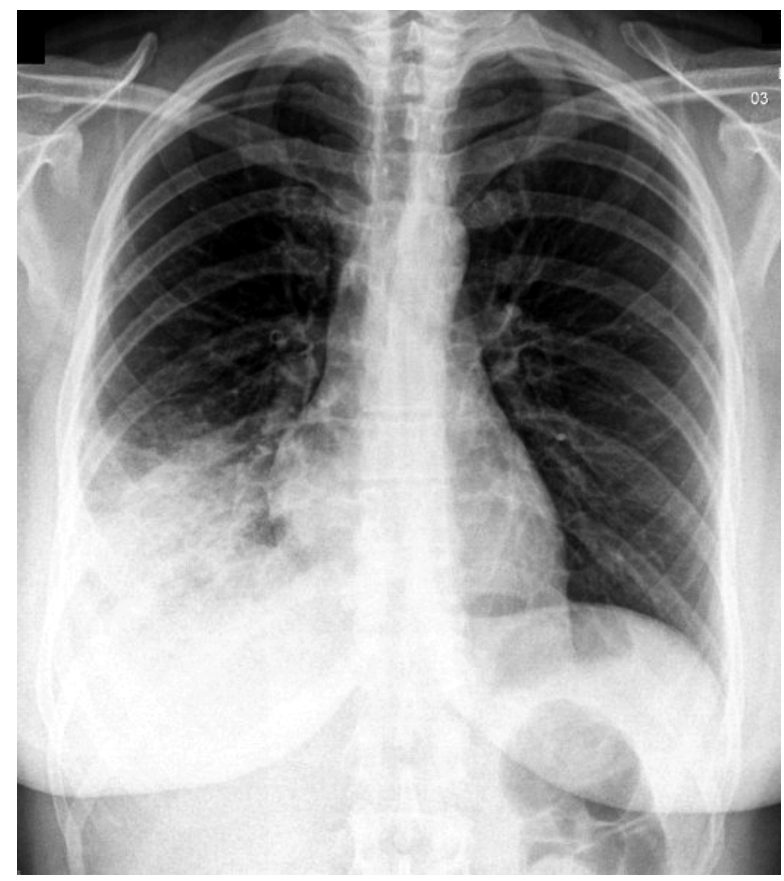

Figure 1. Chest X-ray showing right paracardial consolidation 

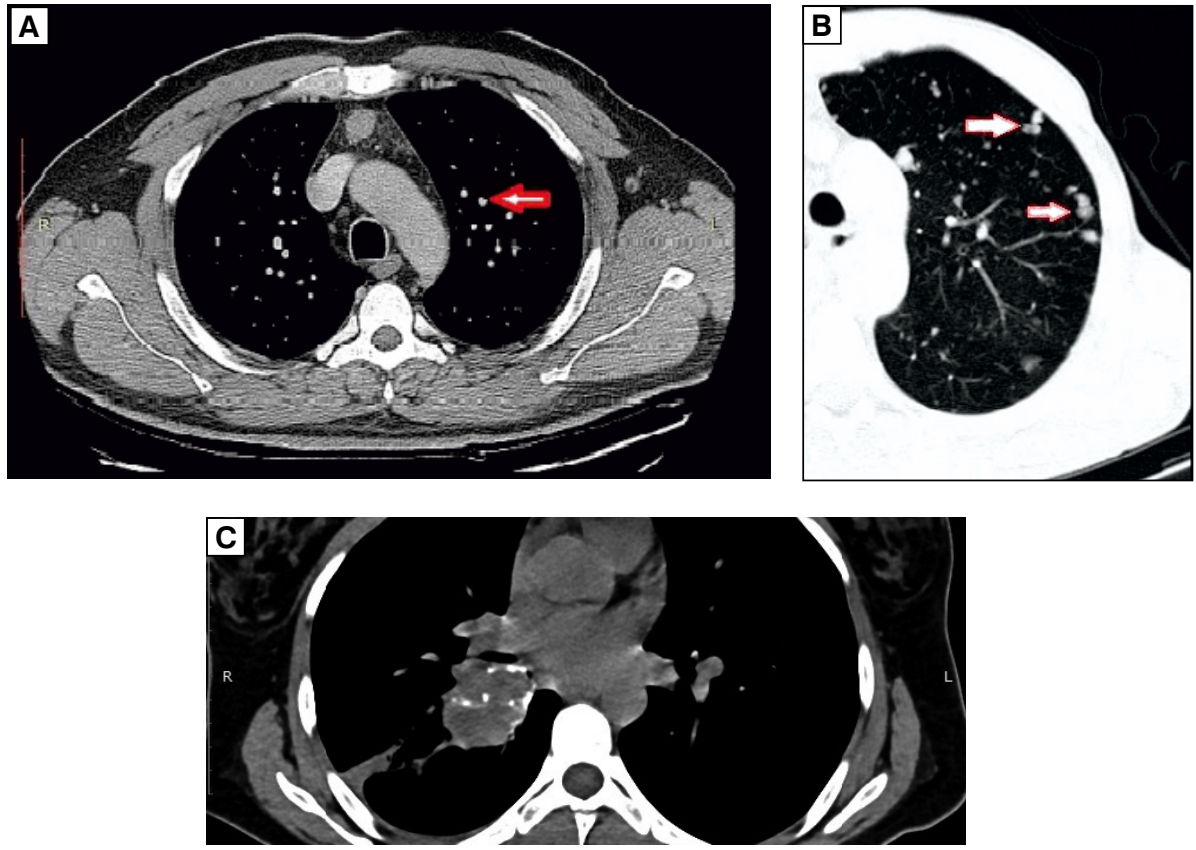

Figure 2. A, B — Lung CT scan presenting diffuse, bilateral, nodules in lung parenchyma; C — large lung infiltrate with central necrosis in right lower lobe
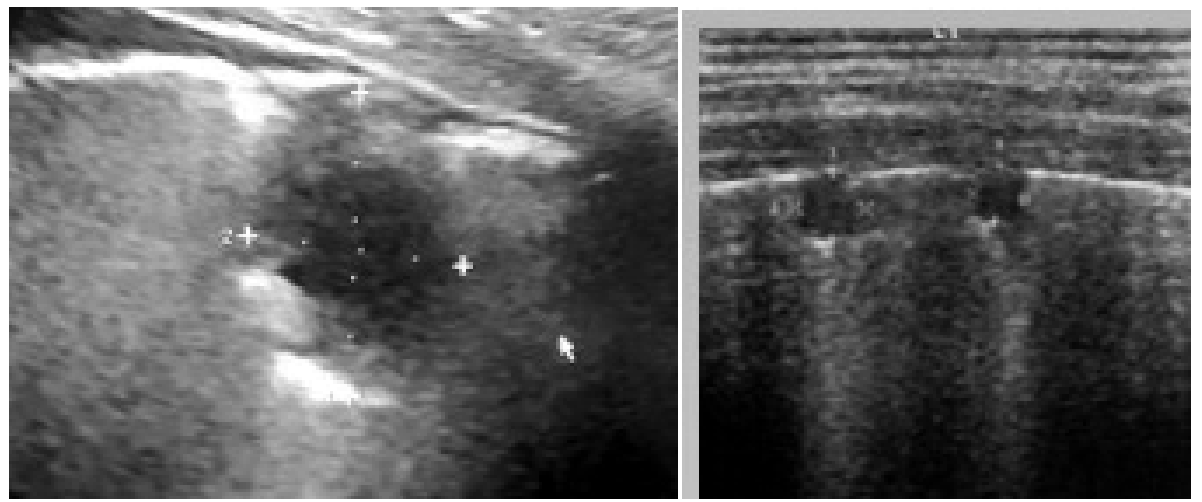

Figure 3. Chest ultrasonography presenting multiple subpleural, hypoechogenic changes with central necrosis

$58 \mathrm{~g} / \mathrm{l}$ (normal range 66-83 $\mathrm{g} / \mathrm{L})$. Urinalysis showed mild proteinuria $(+)$ with a few red blood cell 16-18, epithelial cells $(++)$. Twenty-four-hour urinary protein collection revealed mild proteinuria $0,52 \mathrm{~g} / \mathrm{L}$ (upper limit $0,2 \mathrm{~g} / \mathrm{L}$ ). Gas analyses revealed hypoxemia at the beginning 7.6...8.9...9.3 kPa, hypocapnia at admission $3.7 \ldots 4.2 \ldots 4.6 \mathrm{kPa}$, oxygen saturation $90 \ldots 93 \ldots 95 \%$. Physical examination on admission, blood pressure $120 / 70 \mathrm{mmHg}$, body temperature $38.5^{\circ} \mathrm{C}$, heart rate 122 beats/minute. Swollen ankles, pale skin, conjunctival hyperemia, coagulated blood in both nostrils. Lung auscultation sounds normal, heart rate rhythmic, tachycardic, no murmurs. Abdominal examination without pathological findings. According to anamnesis: cigarette smoker, mother of one child, no allergies reported, no chronic therapy. ECG with sinus tachy- cardia of 122 beats/min, axis normal, no signs of acute heart injury. Lung computed tomography scan (CT) detected diffuse, bilateral, small nodules, some of them with areas of cavitation and pseudo-cavitation and no evidence of thrombosis on CT angiography protocol (Fig. 2). Rheumatologist and nephrologist were consulted and tests for autoimmune disease showed positive c-ANCA $95 \mathrm{U} / \mathrm{ml}$ (normal up to $30 \mathrm{U} / \mathrm{ml}$ ), negative $p$-ANCA, RF $158 \mathrm{IU} / \mathrm{ml}$, antiproteinase 3 value 900 (ref. < 20) (findings suggesting the diagnosis of GPA), kidney function tests and renal ultrasonography normal. IL-6 serum level was normal $5 \mathrm{pg} / \mathrm{ml}$ (ref range 2-5.9 pg/ml), C3 level $1.2 \mathrm{~g} / \mathrm{L}$ (ref range $0.9-1.8 \mathrm{~g} / \mathrm{L}$ ) and C4 level $0.3 \mathrm{~g} / \mathrm{L}$ (ref range $0.1-0.4 \mathrm{~g} / \mathrm{L}$ ). Lung ultrasonography detected bilateral, irregular, subpleural, hypoechogenic changes, with central necrosis (Fig. 3). 

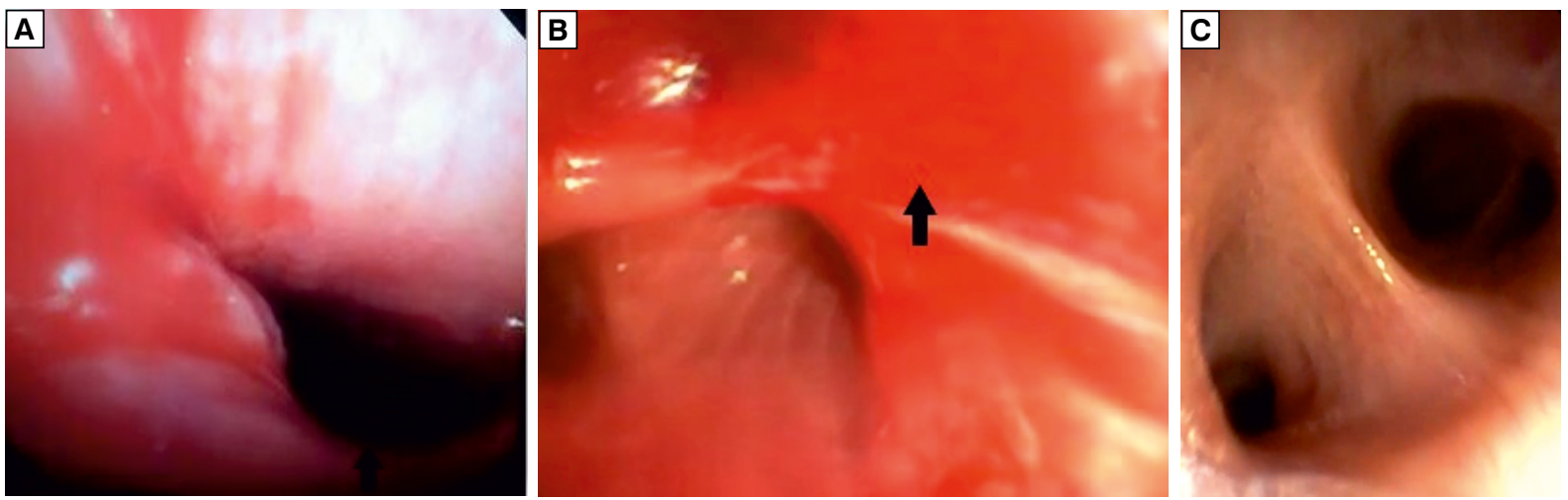

Figure 4. A - Video bronchoscope image presenting edematous, vulnerable respiratory mucosa leading to stenosis of the right intermediate bronchus; B — Video bronchoscope image presenting bleeding of bronchial inflamed mucosa); C - Video bronchoscope image presenting complete resolution of inflamed bronchial mucosa of the right lower lobe)

Echocardiography did not detect pericardial effusion. Mantoux test was negative. Bronchoscopy finding of coagulated blood in both nostrils was the reason for oral intubation. Tracheobronchial mucosa was vulnerable, inflamed and edematous (Fig. 4 A-B). Cytology report of bronchial alveolar lavage $(B A L)$ reported elevated neutrophils. Bronchial lavage was negative for malignancy and infection (microbiology cultivation of BAL specimen and Gene X-pert were negative). Biopsy detected necrotic granulomas with multinucleated giant and inflammatory cells confirming the diagnosis of PGA. The treatment was multidisciplinary and consisted of antipyretic, fluids, wide-spectrum antibiotic, low molecular weight heparin, fluids, vitamins, oxygen, systemic steroids (pulse methylprednisolone followed by oral prednisone prednisolone gradually decreasing the dose) and intravenous cyclophosphamide (Cytoxan) as remission induction therapy. Cyclophosphamide combined with corticosteroids was given four months until the time of remission. There was significant symptom relief, improvement in joint pain and hemoptysis subsided (Fig $4 \mathrm{C}$ ). The corticosteroid dose was slowly reduced and cyclophosphamide was switched to azathioprine (Imuran) to maintain remission. The treatment duration of the maintenance immunosuppressive medication after 10 months follow-up is still ongoing with continuous monitoring of side effects.

\section{Discussion}

ANCAs are typically classified as either myeloperoxidase (MPO)-ANCA, proteinase 3 (PR3)-ANCA and atypical-ANCA. The clinical significance of the two common types or ANCA subsets, MPO-ANCA and PR3-ANCA, differs. MPO-ANCA is often associated with microscopic polyarteritis, necrotizing glomerulonephri- tis and Churg-Strauss syndrome, whereas PR3-ANCA is found primarily in patients exhibiting PGA [11]. There are various infections that have been known to lead to secondary elevation of serum ANCA (tuberculosis, infective endocarditis, hepatitis B), also other autoimmune disorders (rheumatoid arthritis, inflammatory bowel disease, cystic fibrosis, systemic lupus erythematosus, primary sclerosing cholangitis), cancer [11, 12]. Various factors may have an impact on the etiology of GPA which is treated as an autoimmune disease. Genetic factors, infectious agents (like Staphylococcus aureus), environmental factors (like silica, hydrocarbons, fumes, pesticides, and farming) are considered elements for the development of the disease. Mostly, GPA affects the upper and lower respiratory tracts and kidneys and associated with otorhinolaryngological and renal manifestations. However, numerous untypical manifestations may also occur [13]. The American College of Rheumatology established the following classification criteria for GPA: (1) abnormal urinary sediment, [2] abnormal findings on chest radiograph, [3] oral ulcers or nasal discharge, and [4] granulomatous inflammation on biopsy [14]. Here, we have described a case of GPA dominantly presented with lung manifestations (hemoptysis and alveolar hemorrhage). Bronchoscopy as an essential diagnostic in procedure in the evaluation of alveolar hemorrhage, infection, airway disease, and endobronchial lesions, leads to the diagnosis of PGA in our patient. Involvement of the respiratory mucosa can occur along the entire length of the lower and upper airways in $15-55 \%$ of the patients with GPA. In $25 \%$ of the patients with GPA, the involvement of the airways can be the only manifestation [15]. Levels of C3 and C4 were normal, hypocomplementemia with low C3 and/or C4 levels at GPA or MPA diagnosis may be responsible for worse survival and renal prognosis [16]. Platelet to lymphocyte ratio (PLR) as a useful index to estimate the current activity of vasculitis in AAV patients, 
in our patient was 115 , the value $>160$ is associated with poor prognosis [17].

\section{Conclusion}

GPA is a huge challenge for contemporary diagnostics and medicine. High-dose corticosteroids along with other immunosuppressants like cyclophosphamide resulted in significant resolution in our case. Newer therapies are being investigated in order to further improve disease-related outcomes, reducing refractory disease, relapse, chronic organ damage and reduced toxicity.

\section{Conflict of interest: None.}

\section{References:}

1. Holle JU. [ANCA-associated vasculitis]. Internist (Berl). 2015; 56(1): 41-50; quiz 51, doi: 10.1007/s00108-014-3613-8, indexed in Pubmed: 25502657.

2. Panupattanapong S, Stwalley DL, White AJ, et al. Epidemiology and Outcomes of Granulomatosis With Polyangiitis in Pediatric and Working-Age Adult Populations In the United States: Analysis of a Large National Claims Database. Arthritis Rheumatol. 2018; 70(12): 2067-2076, doi: 10.1002/art.40577, indexed in Pubmed: 29806148.

3. Jones RB. Rituximab in the treatment of anti-neutrophil cytoplasm antibody-associated vasculitis. Nephron Clin Pract. 2014; 128(3-4): 243-249, doi: 10.1159/000368580, indexed in Pubmed: 25401382.

4. Yates M, Watts R. ANCA-associated vasculitis. Clin Med (Lond). 2017; 17(1): 60-64, doi: 10.7861/clinmedicine.17-1-60, indexed in Pubmed: 28148583.

5. Berti A Cavalli G, Campochiaro C et al Interleukin-6 in ANCA-associated vasculitis: Rationale for successful treatment with tocilizumab.
Seminars in Arthritis and Rheumatism. 2015; 45(1): 48-54, doi: 10.1016/j.semarthrit.2015.02.002.

6. Binda V, Moroni G, Messa P. ANCA-associated vasculitis with renal involvement. J Nephrol. 2018; 31(2): 197-208, doi: 10.1007/s40620017-0412-z, indexed in Pubmed: 28560688

7. Puéchal $X$. Targeted immunotherapy strategies in ANCA-associated vasculitis. Joint Bone Spine. 2019; 86(3): 321-326, doi: 10.1016/j. jbspin.2018.09.002, indexed in Pubmed: 30201478.

8. Lutalo PMK, D'Cruz DP. Biological drugs in ANCA-associated vasculitis. Int Immunopharmacol. 2015; 27(2): 209-212, doi: 10.1016/j. intimp.2015.04.023, indexed in Pubmed: 25907243.

9. Godse K, Mehta A, Patil S, et al. Omalizumab-A Review. Indian J Dermatol. 2015; 60(4): 381-384, doi: 10.4103/0019-5154.160490, indexed in Pubmed: 26288408

10. Luqmani RA. State of the art in the treatment of systemic vasculitides. Front Immunol. 2014; 5: 471, doi: 10.3389/fimmu.2014.00471, indexed in Pubmed: 25352843.

11. Okauchi S, Tamura T, Kagohashi K, et al. Elevated serum levels of two anti-neutrophil cytoplasmic antibodies in a lung cancer patient: A case report. Biomed Rep. 2016; 5(4): 511-513, doi: 10.3892/br.2016.750, indexed in Pubmed: 27699023.

12. Mahr A, Batteux F, Tubiana S, et al. IMAGE Study Group. Brief report: prevalence of antineutrophil cytoplasmic antibodies in infective endocarditis. Arthritis Rheumatol. 2014; 66(6): 1672-1677, doi: 10.1002/art.38389, indexed in Pubmed: 24497495

13. Miłkowska-Dymanowska J, Laskowska P, Rzuczkowski M, et al. Untypical Manifestations of Granulomatosis with Polyangiitis-A Review of the Literature. SN Comprehensive Clinical Medicine. 2019; 1(8): 616-626, doi: 10.1007/s42399-019-00083-4

14. Leavitt RY, Fauci AS, Bloch DA, et al. The American College of Rheumatology 1990 criteria for the classification of Wegener's granulomatosis. Arthritis Rheum. 1990; 33(8): 1101-1107, doi: 10.1002/art.1780330807, indexed in Pubmed: 2202308.

15. Gupta AS, Mehta AA, Venkitakrishnan R. A case of granulomatosis with polyangiitis (Wegener's granulomatosis) with marked infiltration of Tracheo-bronchial tree. Lung India. 2015; 32(5): 531-532, doi: 10.4103/0970-2113.164172, indexed in Pubmed: 26628780.

16. Deshayes S, Aouba A, Khoy K, et al. PLoS One. 2018 Apr 5. ; 13(4): e0195680.

17. Park HJ, Jung SM, Song JJ, et al. Platelet to lymphocyte ratio is associated with the current activity of ANCA-associated vasculitis at diagnosis: a retrospective monocentric study. Rheumatol Int. 2018; 38(10): 1865-1871, doi: 10.1007/s00296-018-4125-y, indexed in Pubmed: 30088046 